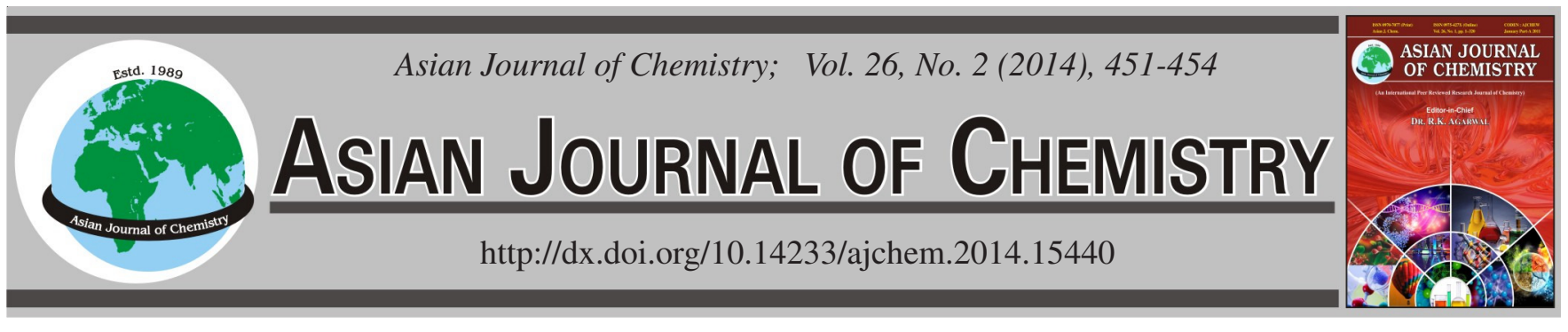

\title{
Effect of Tobacco Stem Treated by Steam Explosion on the Release of Harmful Chemicals in Cigarette Smoke
}

Fang Xue, Dongliang Li*, Guanglin Feng and Guangfu Song

Harmful Components and Tar Reduction in Cigarette Sichuan Key Laboratory/Technology Research Center, China Tobacco Chuanyu Industrial Co. Ltd., Chengdu, P.R. China

*Corresponding author: Fax: +86 28 86005067; Tel: +86 28 86005602; E-mail: cyzygs@163.com; 332425380@qq.com

\begin{abstract}
This paper reported steam explosion technology to treat tobacco stem. The tobacco stem was then added to the tested tobacco shred in appropriate proportion to observe how tobacco stem after steam-explosion treatment affects harmful chemicals in cigarette smoke such as tar, nicotine, CO, phenol and benzo[a]pyrene. The results showed that with the increase of the addition of tobacco stem, the contents of phenol, benzo[a]pyrene, tar and nicotine in cigarette mainstream smoke dropped. Compared with blank samples, the highest drop of phenol, benzo[a]pyrene, tar and nicotine was $36.50,36.63,28.99$ and $38.46 \%$, respectively; On the condition that there was no influence on cigarette burning, the addition of tobacco stem treated by steam explosion can be increased to at most $25 \%$ as tobacco shred substitute.
\end{abstract}

Keywords: Steam explosion, Tobacco stem, Tar, Nicotine, CO, Phenol, Benzo[a]pyrene.

\section{INTRODUCTION}

Steam explosion mainly relies on high temperature and high pressure water to process fibrous materials so as to realize the component separation and structural change of materials through instantaneous release of pressure. At present, steam explosion has already been applied in the research on the pretreatment of materials in paper making, cellulosic ethanol, anaerobic fermentation of straw and animal feed and the biotransformation of lignocellulose ${ }^{1-5}$. In the process of steam explosion, the hemicellulose and lignin in the vegetable fibrous materials produce some acidic substances, degrading part of the hemicellulose to soluble sugar; meanwhile, the lignin in the compound middle lamella softens and is partly degraded, thus weakening the stickiness between fibers ${ }^{6}$.

Tobacco stem accounts for 20-25\% of tobacco leaves and its main components are cell wall materials like lignin, cellulose and hemicellulose. Since its quality is inferior to tobacco leaf, it can produce a large amount of tobacco stem waste in the production of cigarettes. In the burning process, lignin, cellulose and hemicellulose can generate phenol ${ }^{7}$ and benzo[a]pyrene ${ }^{8}$ through pyrolysis or the high temperature synthesis with other components. Phenol can cause intense irritation to human skin and respiratory mucosa and benzo[a]pyrene is highly carcinogenic ${ }^{9,10}$. The nicotine in cigarettes can lead to addiction and also paralyze people's respiratory center or cause cardiovascular diseases ${ }^{11}$. Carbon monoxide can cause harm to human body in that after entering the lung, it goes to the blood quickly and combines with hemoglobin to form carbonyl hemoglobin. This will result disrupt the blood's normal oxygen-carrying capacity, thus leading to hypoxia and anoxemia in the tissue cells. Tar is a carcinogen ${ }^{12}$. It is of great significance to make rational use of this considerable amount of waste to reduce the harmful chemicals in the cigarette mainstream smoke and reduce the health hazard of smoking. This research made a design for the use of steam explosion technology to treat tobacco stem, aiming to raise the utilization rate of tobacco stem while reducing the harmful chemicals in tobacco products such as tar, nicotine, benzo[a]pyrene.

\section{EXPERIMENTAL}

The B2F-grade tobacco stems of flue-cured tobacco were selected in Huili, Sichuan for the year 2009 and the tobacco stem samples underwent the fluidized bed drying on the stem production line. Then the tobacco stems were treated by steam explosion. The B2F-gGrade tobacco stems of flue-cured tobacco were selected in Huili, Sichuan for the year 2009 to blend with the preteated tobacco stems in the proportion of $0 \%$ (blank sample), 5, 10, 15, 20, 25 and $30 \%$, respectively. The blended samples were made into finished cigarettes with cigarette rolling machine.

Acetonitrile (HPLC, Fisher Company); deionized water ( $>5 \mathrm{~m} \Omega$, filtered by $0.45 \mu \mathrm{m}$ membrane); phenol (standard sample, ACROS Company); acetic acid (AR-grade, Sinopharm 
Chemical Reagent Co., Ltd); cyclohexane (HPLC, Fisher Company); benzo[a]pyrene (standard sample, CIL Company); D12-benzo[a]pyrene (internal standard, CIL Company).

RM20/CS-type cigarette-smoking machine (BORGWALD Company, Germany); AX504 analytical balance 0.0001g, Precisa, Swiss); DELTA D200H ultrasonic generator (Delta Company, Taiwan); QB-200 type steam explosion-proof overhead crane (Zhengdao Heavy Machinery Plant, Hebei City, Henan Province); high-performance liquid chromatograph (Agilent 1100$)$; cylinder filter $(0.45 \mu \mathrm{m}$, water system, Tianjin Jinteng Experiment Equipment Co., Ltd), GC-MS (PE Clarus 600); solid-phase extraction column (Varian Company, the U.S.); D49-type comprehensive test bench (SODIM Company, France).

Steam explosion pre-treatment of tobacco stem: This research used saturated steam as the working medium. The working pressure was $0.6 \mathrm{MPa}$ and the steaming time was $20 \mathrm{~s}$. After the steam explosion experimental facility reached the working pressure, we carried out 4-5 times of air explosion, then preheated the system. The tobacco stem samples were added for steam explosion pretreatment.

Detection of phenol: We tested the release amount of phenol in the cigarette mainstream smoke according to $\mathrm{YC} / \mathrm{T}$ 255-2008 standard method ${ }^{13}$ to determine the release amount of phenol in the cigarette mainstream smoke.

Benzo[a]pyrene detection: We tested the release amount of benzo[a]pyrene in the cigarette mainstream smoke according to GB/T 21130-2007 standard method ${ }^{14}$.

Sensory evaluation method: The finished cigarette samples were screened and balanced based on certain physical parameters. Then we took 40 testing cigarettes and 40 blank control cigarettes (the addition of tobacco stem was $0 \%$ ), which was evaluated by evaluation staff on the nine-point-scale for unblended cigarettes.

TABLE-1

EVALUATED INDICATORS FOR UNBLENDED CIGARETTES

\begin{tabular}{ccc} 
Aroma property & Smoke property & Flavor property \\
\hline Aroma quality & Impact & Irritation \\
Smoke amount & Fineness & Dry sensation \\
Foreign smoke & Concentration & Cleanness \\
Diffusion & Clustering & Sweetness \\
\hline
\end{tabular}

Detection of smoke chemicals: The contents of total particulate matter, tar, carbon monoxide and nicotine in the mainstream smoke of cigarette with the addition of different proportions of tobacco stems were detected according to the reported methods ${ }^{15,16}$.

Detection of chemicals in mainstream smoke: The detection of cellulose, hemicellulose and lignin in the cell wall of tobacco stem before and after steam explosion was carried out with the methods reported in literature ${ }^{17}$. The detection of potassium, chlorine, total sugar and protein was carried out with the method stipulated by tobacco industry ${ }^{18,19}$.

\section{RESULTS AND DISCUSSION}

Analysis of the release amount of phenol in cigarette smoke based on different proportions of tobacco stems treated by steam explosion: We detected the release amount of phenol in the testing samples containing different proportion of tobacco stems by the reported method ${ }^{13}$. The results are shown in Fig. 1.

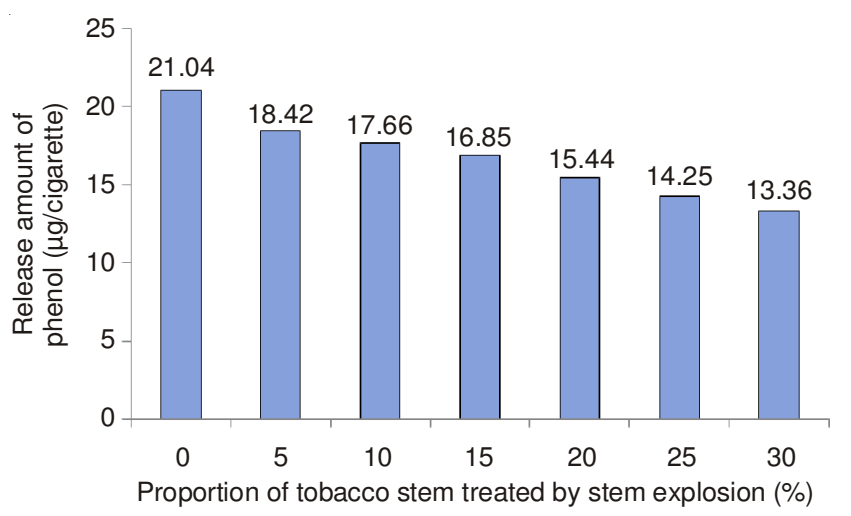

Fig. 1. Influence of different proportions of tobacco stems on the release amount of phenol in the mainstream smoke of cigarettes

Fig. 1 showed that the cigarettes with no tobacco stems had the highest release amount of phenol, reaching $21.04 \mu \mathrm{g} /$ cigarette. The cigarettes with the addition of tobacco stems showed a significant drop in the release amount of phenol; with the increase in the addition, the release amount of phenol in corresponding cigarette samples gradually dropped and reached the lowest value when the proportion was $30 \%$. Compared with the blank sample (no addition of tobacco stem), when the addition of tobacco stems reached $30 \%$, the maximal drop in the release amount of phenol was $36.50 \%$. This may be because steam explosion technology can reduce the cellulose, hemicellulose and lignin in the tobacco stems, which are important precursors for producing phenol. Therefore, steam explosion technology reduces the release of phenol in the tobacco stems.

Analysis of the release amount of benzo[a]pyrene in cigarette smoke based on different proportions of tobacco stems treated by steam explosion: The release amount of benzo[a]pyrene in the testing samples containing different proportion of tobacco stems was detected by the reported method $^{14}$. The results are shown in Fig. 2.

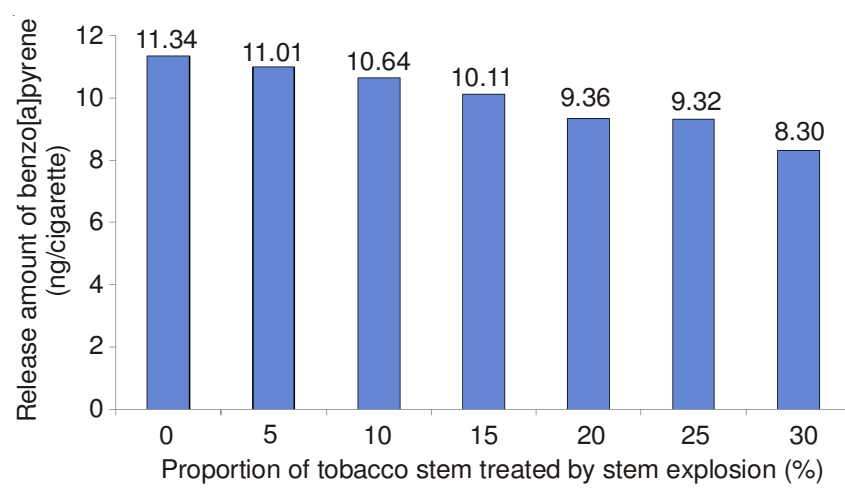

Fig. 2. Influence of different proportions of tobacco stems treated by steam explosion on the release amount of benzo[a]pyrene in the mainstream smoke of cigarettes

Fig. 2 showed that the cigarettes without any tobacco stems showed the highest level of benzo[a]pyrene, which reached $11.34 \mathrm{ng} /$ cigarette. The cigarettes with the addition of tobacco stems showed a significant drop in the release amount of 
benzo[a]pyrene. With the increase in the addition, the release amount of phenol in the corresponding cigarette samples gradually dropped and reached the lowest value when the proportion was $30 \%$. Compared with blank samples (the proportion of tobacco stem was $0 \%$ ), when the proportion of tobacco stems was $30 \%$, the maximal drop in the release amount of benzo[a]pyrene could reach $36.63 \%$.

Influence of the different proportions of tobacco stems treated by steam explosion on the sensory quality of cigarettes: We carried out evalution by the method discussed previously on the cigarettes containing different proportions of tobacco stems treated by steam explosion (Table-2).

TABLE-2

INFLUENCE OF DIFFERENT PROPORTIONS OF TOBACCO STEMS ON THE SENSORY QUALITY OF CIGARETTES

\begin{tabular}{ccccc}
\hline $\begin{array}{c}\text { Proportion } \\
\text { of addition }\end{array}$ & $\begin{array}{c}\text { Aroma } \\
\text { property }\end{array}$ & $\begin{array}{c}\text { Taste } \\
\text { property }\end{array}$ & $\begin{array}{c}\text { Smoke } \\
\text { property }\end{array}$ & $\begin{array}{c}\text { Total } \\
\text { points }\end{array}$ \\
\hline 0 & 24.00 & 24.00 & 23.00 & 71.00 \\
5 & 24.25 & 23.00 & 24.00 & 71.25 \\
10 & 24.25 & 23.00 & 24.00 & 71.25 \\
15 & 23.75 & 21.75 & 23.75 & 69.25 \\
20 & 23.75 & 22.50 & 23.75 & 70.00 \\
25 & 25.75 & 23.50 & 24.00 & 73.25 \\
30 & 23.75 & 21.75 & 24.00 & 69.50 \\
\hline
\end{tabular}

Table-2 showed that the smoke of cigarettes containing different proportions of tobacco stems had improved, but only to a limited extent. There was no noticeable change in the overall aroma property, while the flavor property showed slight decline. Analyzed from the overall change trend, when the proportion of tobacco stems rose from 5 to $30 \%$, the testing samples could preserve good sensory quality, with no obvious changes. When the content of tobacco stems was $25 \%$, the testing samples showed the best sensory quality and when the addition of tobacco stems reached $30 \%$, the sample's sensory quality began to decline. The following are the possible causes of the variation of sensory quality. First of all, the main chemical compositions of tobacco stem are cellulose, hemicellulose, lignin and other cell wall materials, which are related to the irritation of cigarettes ${ }^{11,20}$, steam explosion can effectively destroy the fibrous structure of the tobacco stem, reducing their content and thus reducing the adverse impact on cigarettes' sensory quality.

Influence of different proportions of tobacco stems treated by steam explosion on common chemicals in the mainstream smoke of cigarettes: We carried out detection on the smoke components in the testing samples with different proportions of tobacco stems, including total particulate matter, tar, carbon monoxide and nicotine by reported methods ${ }^{15,16}$. The results are shown in Table- 3 .

According to Table-3, with the increase of the addition amount of tobacco stems, the contents of common smoke components like total particulate matter, tar and nicotine showed a declining trend, while the content of carbon monoxide remained largely unchanged. Compared with blank samples (the addition of tobacco stems was $0 \%$ ), the largest drop in total particulate matter, tar and nicotine was 28.16, 28.99 and $38.46 \%$, respectively. Increased proportion of tobacco stems cannot only reduce the amounts of tar and

\begin{tabular}{ccccc}
\hline \multicolumn{5}{c}{ TABLE-3 } \\
INFLUENCE OF DIFFERENT PROPORTIONS OF \\
TOBACCO STEMS TREATED BY STEAM EXPLOSION \\
ON SMOKE PROPERTY OF CIGARETTES
\end{tabular}

nicotine in the cigarette smoke, but is also beneficial to the health of smokers. Therefore, the application value of tobacco stems is confirmed. The addition of tobacco stems could also bring down the production cost.

Influence of different proportions of tobacco stems on the release of potassium-chloride ratio in cigarettes: Potassium-chloride ratio is the major indicator influencing the performance of tobacco shred. High potassium-chloride ratio can improve the performance of tobacco shred, resulting in excellent aroma and flavor ${ }^{21}$. Fig. 3 shows the influence of different proportions of tobacco stems on potassium-chloride ratio in cigarette samples.

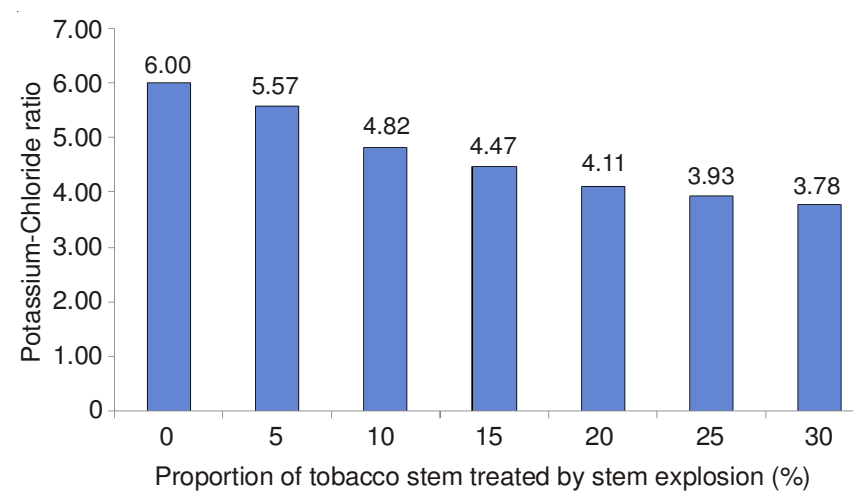

Fig. 3. Influence of different proportions of tobacco stem on potassiumchloride ratio in cigarettes

Fig. 3 shows that as the addition of tobacco stems increased from 5 to $30 \%$, the potassium-chloride ratio in the testing samples declined compared with the control samples (the addition amount of tobacco stems was $0 \%$ ) and there was an overall trend of decrease. When the content of tobacco stems reached $30 \%$, the ratio would show the highest drop, reaching $37 \%$. In the single-index evaluation, the potassium-chloride ratio in the testing samples stayed above 3.7 , indicating that the tobacco shred in various testing samples had good combustibility. When the addition amount of tobacco stems increased from 0 to $30 \%$, the relatively high potassium-chloride ratio of testing samples was consistent with their high level of sensory quality.

Influence of different proportions of tobacco stems on the Shmuck value of cigarettes: Shmuck value measure the balance and coordination between the flavor and irritation of the smoke released by tobacco shred and the acid-base content. In this test, we observed the influence of different addition 
amounts of tobacco stems treated by steam technology on the Shmuck value in cigarettes samples and the results are shown in Fig. 4.

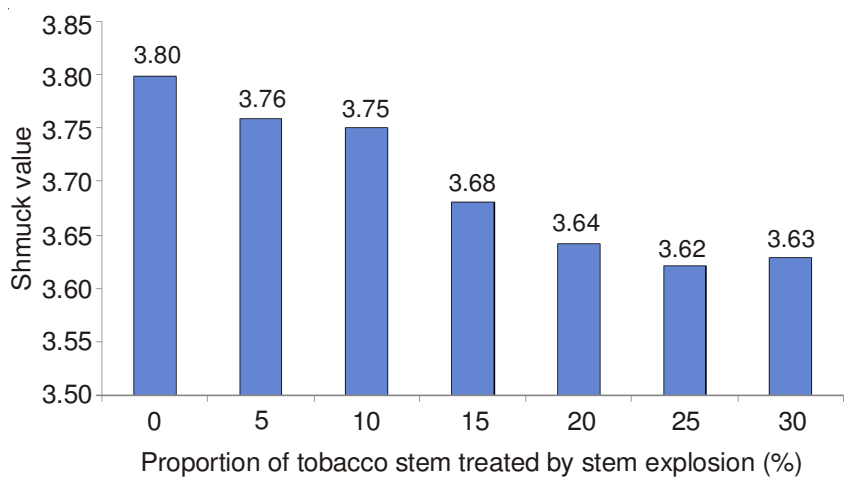

Fig. 4. Influence of different addition amounts of tobacco stem on Shmuck value of cigarettes

The Shmuck value in tobacco industry, which is used to characterize the quality of tobacco, is the content ratio of total water-soluble sugar to protein. It is generally believed that ideally, Shmuck value should be within 2 and the value exceeding 2 would mean certain limitation in the evaluation. That is because excessive sugar content can disrupt the balance and coordination, making cigarette smoke plain and tasteless and lose the original smoke strength ${ }^{21}$. In this test, it can be seen from the analysis of the testing samples that the Shmuck value of control samples (the pure tobacco shred with $0 \%$ addition of tobacco stems treated by steam explosion) was maintained at 3.80. As the addition amount of tobacco stems increased from 5 to $30 \%$, the Shmuck value in both the testing samples and control samples was declining. When the content of tobacco stems reached $25 \%$, the ratio showed the biggest drop, reaching $4.74 \%$.

Analysis of the influence of steam explosion pre-treatment on cellulose, hemicellulose and lignin in the cell wall materials of tobacco stems: It is currently believed that cell wall materials are sugars that can have an adverse effect on the qualities and flavor of tobacco. This is because the pyrolysis of these materials can produce a large amount of low-level aldehydes, which may cause irritable cough. The changes in the contents of different cell wall materials in tobacco stem samples before and after the steam explosion are shown in Table-4.

TABLE-4

CONTENTS OF CELL WALL MATERIALS IN TOBACCO STEM SAMPLES BEFORE AND AFTER STEAM EXPLOSION

\begin{tabular}{cccc}
\multicolumn{4}{c}{ SAMPLES BEFORE AND AFTER STEAM EXPLOSION } \\
\hline \multirow{2}{*}{ Serial number } & $\begin{array}{c}\text { Hemicellulose } \\
(\%)\end{array}$ & $\begin{array}{c}\text { Cellulos } \\
(\%)\end{array}$ & $\begin{array}{c}\text { Lignin } \\
(\%)\end{array}$ \\
\hline Before explosion & 10.61 & 29.87 & 5.10 \\
After explosion & 8.94 & 24.65 & 5.05 \\
\hline
\end{tabular}

According to Table-4, after steam explosion, the contents of cellulose and hemicellulose showed significant drop compared with to that before, that is, by 15.74 and $17.48 \%$, respectively, while there was no significant change in the content of lignin. Therefore, the use of steam explosion technology in treating tobacco stems in this test can promote the degradation of cellulose and hemicellulose in the cell wall materials of tobacco stems, thus alleviating the discomfort occurring when smoking and improving the quality. This can demonstrate from another perspective that when the addition amount of tobacco stems in the test increased to $25 \%$, the test samples could still maintain good smoking quality.

\section{Conclusion}

The test mainly studied the effect of adding tobacco stems after steam explosion pre-treatment on the release of such harmful chemicals as phenol, benzo[a]pyrene, tar, carbon monoxide and nicotine in the mainstream smoke of finished cigarettes, The results showed that the addition of the tobacco stems after steam explosion pre-treatment can significantly reduce the contents of phenol, benzo[a]pyrene, tar and nicotine in the mainstream smoke of cigarettes; with the increase of addition of the tobacco stems, the contents of phenol, benzo[a]pyrene, tar and nicotine in the mainstream smoke of cigarettes reduced. Compared with blank samples, the biggest drop of phenol, tar and nicotine could reach 36.50, $36.63,28.99$ and $38.46 \%$, respectively. On the condition that there is no impact on the sensory quality and the burning of cigarettes, at most $25 \%$ of steam-explosion tobacco stem can be added as substitute for tobacco shred.

\section{REFERENCES}

1. R.S. Martin, C. Perez and R. Briones, Bioresour. Technol., 217 (1995).

2. L.H. Zhang, D. Li, L.J. Wang, T.- Wang, L. Zhang, X.D. Chen and Z.Mao, Bioresour. Technol., 8512 (2008).

3. W. Xu, G. Ke, J. Wu and X. Wang, Eur. Polym. J., 42, 2168 (2006).

4. H. Kimt, F. Taylor and K.B. Hicks, Bioresour. Technol., 99, 5694 (2008).

5. S.Y. Jin and H.Z. Chen, Process Biochem., 42, 188 (2007).

6. C. Garcia-Jaldon, D. Dupeyre and M.R. Vignon, Biomass Bioenergy, 251 (1998).

7. W.S. Schlotzhauer and O.T. Chortyk, Tobacco Sci., 25, 6 (1981).

8. W.S. Schlozhauer and O.T. Chortyk, J. Anal. Appl. Pyrol., 193 (1987).

9. Y.L. Zhang, Cigarette Tar Content Reduction Engineering [M], China Light Industry Press, Beijing (2000).

10. J.P. Xie, Evaluation Principles and Methods of Cigarette Perniciousness, Chemical Industry Press, Beijing (2009).

11. K.Y. Yan, Chemisity of Tobacco Stem, Zhengzhou University Press, Zhengzhou, China (2002).

12. X.Y. Zhan, D.D. Song, Y. Yu and J.L. Li, Environ. Pollut. Control, 25, 142 (2003).

13. Industrial Standard of P.R. China, YC/T 255-2008, Cigarettes-Determination of Major Phenolic Compounds in Mainstream Cigarette Smoke-High Performance Liquid Chromatographic Method.

14. Standard of P.R. China, GB/T 21130-2007, Cigarettes-Determination of Benzo[a]pyrene in Total Particulate Matter.

15. Standard of P.R. China, GB/T 23355-2009, Cigarettes-Determinations of Nicotine in Smoke Condensates-Gas Chromatographic Method.

16. Standard of P. R. China, GB/T 23356-2009,Cigarettes-Determination of Carbon Monoxide in the Vapour Phase of Cigarette Smoke-NDIR Method.

17. X.Y. Guo, Y.H. Dai, Z.M. Guo and M.J. Wu, Tobacco Sci. Technol., 1, 43 (2009).

18. Standard of P.R. China, YC/T173-2003, Tobacco and Tobacco ProductsDetermination of Potassium-Flame Photometry.

19. Standard of P.R. China, YC/T249-2008, Tobacco and Tobacco ProductsDetermination of Protein-Continuous Flow Method.

20. Y.X. Wang, S.L. Ge and T. Jia, Tobacco Sci. Technol., 3, 16 (1996).

21. R.X. Wang, Tobacco Chemistry, Chinese Agriculture Press, Beijing (2003). 